\title{
Handwashing Practices in Neonatal Intensive Care Unit, Paediatric Intensive Care Unit and Neonatal Nurseries in Patan Hospital
}

\author{
Suchita Joshi, ${ }^{1}$ Puja Amatya, ${ }^{1}$ Bibek Poudel, ${ }^{1}$ Saroj Adhikari Yadav ${ }^{1}$ \\ ${ }^{1}$ Patan Academy of Health Sciences, Lalitpur, Nepal.
}

\begin{abstract}
Background: Hand hygiene has been identified as the single most important factor in minimising hospital acquired infections. However, compliance of handwashing guidelines has remained low. The aim of this study was to study the handwashing practices in the Paediatric and Neonatal intensive care units and Neonatal nurseries in Patan Hospital, and secondly to re-evaluate the improvement on compliance of handwashing guidelines after intervention.

Methods: Pre-intervention study was conducted by covertly observing the handwashing practices by the healthcare workers. The healthcare workers were then shown the video demonstrating correct methods of handwashing as recommended by World health organization. The cycle was completed by discretely re-observing the handwashing practices following intervention.

Results: Sixty five samples were collected initially. Only $6(9.2 \%)$ had completed all steps of handwashing correctly. Post- intervention, 51 samples were collected, out of which $35(68.6 \%)$ had correctly completed all the steps. Following audio-visual demonstration, $100 \%$ correctly completed 8/10 steps of handwashing with soap and water. $8(16 \%)$ failed to dry hands using a single use towel and $14(28 \%)$ failed to turn off the tap using elbow. Postintervention, $100 \%$ correctly completed $4 / 7$ steps of handwashing using chlorhexidine. Four (15\%) still failed to rub backs of fingers to opposite palm, eight (30\%) failed to palm to palm with fingers interlaced, and rub thumb to opposite palm.

Conclusions: Compliance in hand hygiene is low despite the known fact that it reduces nosocomial infections. However, a simple intervention like video demonstration can improve the compliance among healthcare workers.

Keywords: Disinfection; handwashing; infection control; nosocomial infection.
\end{abstract}

\section{INTRODUCTION}

Hospital acquired infection has been identified as a major cause of death ${ }^{1}$ and prolonged hospital stay for patients thereby increasing the financial burden..$^{2-4}$ Hands of healthcare workers have been identified as the most important factor in hospital acquired infections. ${ }^{5}$ It is also well established that this can be minimised by appropriate handwashing. ${ }^{6-8}$ However, hand hygiene compliance among healthcare workers have been shown to be sub-optimal.9,10 The significance of nosocomial infection is more in the paediatric and neonatal population because of their immature immunity.
World Health Organisation (WHO) ${ }^{11}$ and Centre for Disease Control $(C D C)^{12}$ have introduced guidelines for proper handwashing by healthcare workers to improve handwashing practices and hence to minimise hospital acquired infections.

The aim of this study was to study the handwashing practices among healthcare workers in the Paediatric and Neonatal intensive care units and Neonatal nurseries in Patan Hospital, and secondly to re-evalute the improvement on compliance of handwashing guidelines following intervention. 


\section{METHODS}

This is a cross-sectional quality improvement audit. To assess the compliance of handwashing guidelines among the healthcare workers, handwashing practices of the doctors and nurses working in the PICU, NICU and neonatal nurseries were discreetly observed by members of the research team. The healthcare workers were unaware of the ongoing study. Data were collected for a one month period (October to November 2015). The observers noted if the healthcare workers followed each of the steps of handwashing as recommended by WHO while washing hands with soap and water, or chlorhexidine solution.

Following the primary study, all the doctors and nurses working in the PICU, NICU and the neonatal nurseries were shown the video demonstrating correct steps of handwashing using soap and water, and with chlorhexidine gel. The open access audio-visual tool prepared by "WHO collaborating centre for Training and Research in Newborn Care, AlIMS, New Delhi" was used. Re-evaluation was carried out a week after all healthcare workers had the opportunity to watch the video. The healthcare workers were once again unaware of the re-evaluation.

The pre and post intervention data were analysed using "Microsoft Excel: mac 2011" to observe if there was improvement in the handwashing practices among the healthcare workers after watching the video.

Ethical approval for this study was taken from the Institutional Review Committee (IRC), Patan Academy of Health Sciences.

\section{RESULTS}

A total of 65 healthcare workers were observed while they were using soap and water, and chlorhexidine solution to wash hands for pre intervention data in the primary audit, out of which only six (9.2\%) had thoroughly completed all the steps of hand washing. Similarly, 51 healthcare workers were observed in post-intervention re-audit out of which 35 (68.6\%) had thoroughly completed all steps of hand washing.

When hand washing was done with soap and water during pre-intervention primary audit, none out of 21 healthcare workers washed hands using soap and water completing all steps of hand washing (Figure 1). Healthcare workers were good at wetting hands properly $(21,100 \%)$, applying adequate soap $(19,90.5 \%)$ and rubbing palm to palm $(19,90.5 \%)$. Subjects were worse at washing with back of fingers to palms with fingers interlocked $(3,14.3 \%)$, rotational rubbing of clasped fingers $(6,28.6 \%)$ and using elbow to turn off the tap (7, 33.3\%) (Figure 2).

After audio-visual demonstration of the correct steps of handwashing all 51 (100\%) correctly completed 8/10 steps of handwashing with soap and water. Eight (16\%) failed to dry hands using a single use towel and 14 (28\%) failed to turn off the tap using elbow (Figure 2).

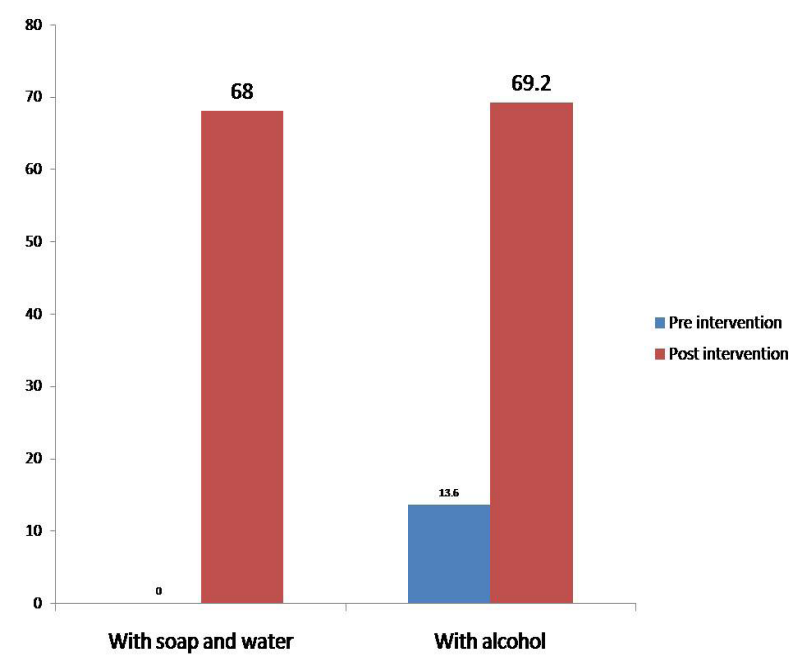

Figure 1. Percentage of hand washing completed correctly completing all steps during preintervention primary and post-intervention.

When hand washing was done with chlorhexidine solution during pre-intervention primary audit, only 6 / 44 (13.6\%) washed hands completing all the steps of hand washing. As shown in Figure 3, rubbing of palm to palm and fingers to fingers including webs were both done correctly 30 (68.2\%) and 28 (63.6\%) times, respectively. But rubbing knuckle to palm and rubbing the thumbs both were done correctly only 11 times (25\% each) and rubbing knuckle to palm was done only 14 times $(31.8 \%)$.

When hand washing was done with chlorhexidine during post- intervention, 18 (69.2\%) out of 26 had washed hands completing all the steps of hand washing with chlorhexidine. All 41 (100\%) applied enough solution, rubbed palm to palm and palm over opposite dorsum as well as rubbed the knuckles over opposite palm. One each failed to rub backs of fingers to opposite palm, and rubbing thumbs to palm. Six failed to rub palm to palm with fingers interlaced (Figure 3). 

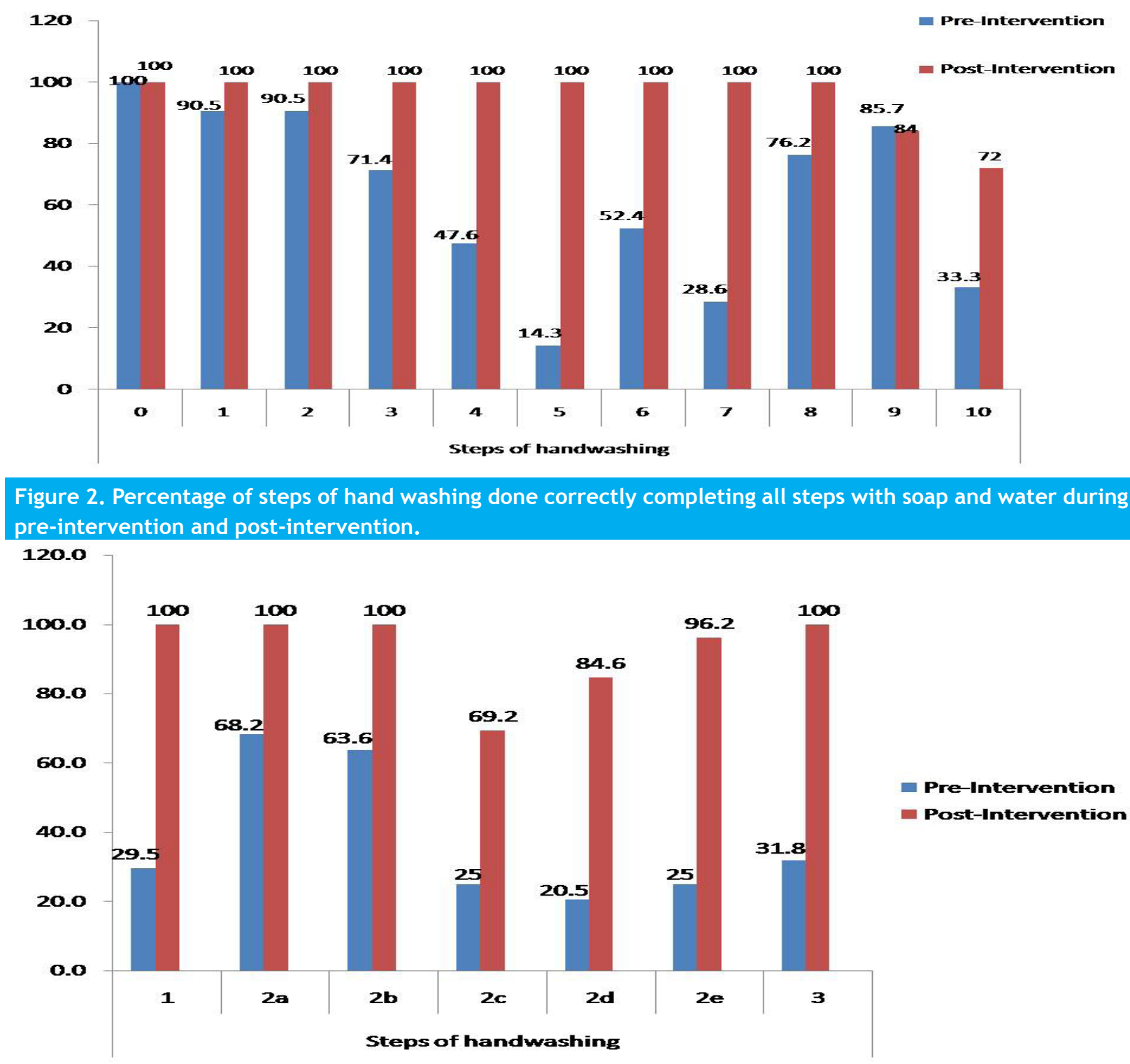

Figure 3. Percentage of steps of hand washing done correctly completing all steps with alcohol during preintervention and post-intervention.

\section{DISCUSSION}

This audit was carried out on healthcare workers working in areas where there is high risk for nosocomial infection. The neonatal units in our hospital have been known to suffer outbreaks of multidrug resistant organisms in the past.${ }^{13}$ Therefore, the healthcare workers in these units are well aware of the importance of handwashing to prevent hospital acquired infection. Despite that, our primary audit showed that the compliance was suboptimal. This reflects the condition globally. ${ }^{14,15}$

Our pre-intervention primary audit showed that healthcare workers miss following certain steps of handwashing, such as rubbing backs of fingers, knuckles and webs of fingers when washing hands with soap and water or with chlorhexidine solution. After washing hands with soap and water, healthcare workers did not always use the single use paper towels to dry their hands. When washing hands with chlorhexidine solution, many were using inadequate amount of the solution. This may have been due to inadequate knowledge. Therefore, we chose audio-visual demonstration of the correct steps of handwashing using both soap and water, and chlorhexidine solution as our method of intervention. 
Different interventions such as educational program, ${ }^{16}$ routine surveillance ${ }^{17}$ and gaming techniques ${ }^{18}$ have been tried to improve compliance of handwashing practices among healthcare workers. Each of these interventions has shown to improve the compliance of hand hygiene among healthcare workers. It was reassuring to see that the intervention that we used also produced the desired results. After watching the video, 8/10 steps of handwashing using soap and water was performed correctly by all. Similarly, 4/7 steps of handwashing using chlorhexidine solution were performed correctly. This showed that reminding the healthcare workers of the correct methods of hand hygiene by audio-visual aids and be effective in increasing compliance.

Epidemiological evidence has shown that hand hygiene is effective in reducing healthcare associated infection but compliance with hand hygiene has been historically found to be poor. ${ }^{11} \mathrm{~A}$ systematic review on hand hygiene interventions carried out in 2010 has also demonstrated that $18 / 31$ (58\%) studies included in the review showed significant improvement in healthcare associated infection after intervention related to hand hygiene among HCW. ${ }^{19}$ As a cost effective and highly effective method of infection control, it is vital that correct handwashing methods and its compliance be implemented in our hospitals. Our study has demonstrated that active surveillance and reminding the HCW of the correct method of hand hygiene can be effective. However, to ensure that this improvement is sustained, it may be necessary to continue training and active surveillance.

\section{CONCLUSION}

This study suggests that audio-visual demonstration can improve compliance of proper hand washing technique. Sharing knowledge on need and importance of hand hygiene and proper steps of hand washing improves the performance and motivation. Therefore, we suggest regular surveillance and audio-visual demonstration of handwashing technique to all healthcare workers to improve compliance.

\section{REFERENCES}

1. Hitrz DG, Nelson KB. The natural history of febrile seizures. Annu Rev Med. 1983;34(1):453-71.

2. Østergaard JR. Febrile seizures. Acta Paediatr. 2009;98(5):771-3.
3. Singhi PD, Srinivas M. Febrile seizures. Indian Paediatr. 2001;38:733-40.

4. Pavlidou E, Tzitiridou M, Kontopoulos E, Panteliadis CP. Which factors determine febrile seizure recurrence? A prospective study. Brain Dev. 2008;30(1):7-13.

5. Ojha AR, Shakya KN, Aryal UR. Recurrence Risk of Febrile Seizures in Children. J Nepal Paediatr Soc. 2012;32(1):336.

6. Berg AT, Shinnar S, Hauser WA, Leventhal JM. Predictors of recurrent febrile seizures: a metaanalytic review. J Pediatr. 1990;116(3):329-37.

7. FukuyamaY, SekiT, Ohtsuka C, Miura H, Hara M. Practical guidelines for physicians in the management of febrile seizures. Brain Dev. 1996;18(6):479-84.

8. Farrell K, Goldman RD. The management of febrile seizures. BCMJ. 2011;53(6):268-73.

9. Millichap JJ, Millichap JG. Method of investigation and management of infections causing febrile seizures. Paediatr Neurol. 2008;39(6):381-6.

10. Mustafić N, Tahirovi ć H, Trnovcević J, Kapidzić A. Clinical characteristics at onset of first febrile convulsions. Acta Med Croatica. 2008;62(5):511-5.

11. Trainor JL, Hampers LC, Krug SE, Listernick R. Children with first time simple febrile seizures are at low risk of serious bacterial illness. Acad Emerg Med. 2001;8(8):7817.

12. Bessisso MS, Elsaid MF, Almula NA, Kadomi NK, Zeidan SH, Azzam SB, et al. Recurrent risk after a first febrile convulsion. Saudi Med J. 2001;22(3):254-8.

13. Zhao F, Emoto SE, Lavine L, Nelson KB, Wang CC, Li $\mathrm{SC}$, et al. Risk factors for febrile seizures in the People's Republic of China: a case control study. Epilepsia. 1991;32(4):510-4.

14. Van Stuijvenberg M, Steyerberg EW, Derksen-Lubsen G, Moll HA. Temperature, age and recurrence of febrile seizure. Arch Pediatr Adolesc Med. 1998;152(12):1170-5.

15. Al-Eissa YA. Febrile seizures: rate and risk factors for 
Handwashing Practices in Neonatal Intensive Care Unit, Paediatric Intensive Care Unit and Neonatal Nurseries

recurrence. J Child Neurol. 1995;10(4):315-9.

16. Knudsen FU. Recurrence risk after first febrile seizure and effect of short term diazepam prophylaxis. Arch Dis Child. 1985;60(11):1045-9.
17. Pavlidou E, Tzitiridou M, Ramantani G, Panteliadis C. Indications for intermittent diazepam prophylaxis in febrile seizures. Klin Padiatr. 2005;218(5):264-9. 